\title{
Prediabetes after kidney transplantation-a reason to worry?
}

\author{
Trond G. Jenssen ${ }^{1,2}$
}

As many as $10 \%$ to $20 \%$ of patients undergoing kidney transplantation develop post-transplant diabetes mellitus, which is associated with increased mortality. Even borderline changes in glucose metabolism, so-called prediabetes, may involve a similar risk. A recent study by Porrini et al. showed for the first time that such changes in glucose metabolism are in fact associated with future cardiovascular disease and death in kidney-transplanted patients. This commentary discusses the relevance and clinical implications of these new findings.

Diabetes mellitus diagnosed after organ transplantation is defined as post-transplant diabetes mel-

litus (PTDM) as opposed to type 2 diabetes, which is present before trans- plantation and is the most common form of diabetes in the general popu- lation. Both conditions are diagnosed according to the World Health Organi- zation glucose criteria defined by an oral glucose tolerance test (OGTT). However, the 2 conditions differ in many ways: (i) the occurrence of PTDM is largely driven by a chemical mecha- nism due to immunosuppressant drugs in addition to common genetic predis- position and lifestyle factors as seen in type 2 diabetes; (ii) a threshold level of hemoglobin $\mathrm{A}_{1 \mathrm{c}} \quad\left(\mathrm{HbA}_{1 \mathrm{c}}\right)$ is established to diagnose type 2 diabetes, but such a threshold level of $\mathrm{HbA}_{1 \mathrm{c}}$ is still not established for the diagnosis of PTDM ${ }^{1}$; and (iii) in type 2 diabetes, glucose (and $\mathrm{HbA}_{1 c}$ ) criteria for the diagnosis of type 2 diabetes are established according to

\footnotetext{
$\mathrm{a}$

${ }^{1}$ Department of Transplantation, Oslo University Hospital, Oslo, Norway; and IInstitute of Clinical Medicine, University of Oslo, Oslo, Norway
}

Correspondence: Trond G. Jenssen, Department of Transplantation, Oslo University Hospital, Sognsvannsveien 20, Oslo 0372, Norway. E-mail: tjenssen@ous-hf.no

the risk of developing microvascular complications, especially retinopathy. ${ }^{2}$ The major concern in patients with PTDM is, however, not the risk of microvascular disease, but rather the risk of cardiovascular disease with sub- sequent death.

After kidney transplantation, $10 \%$ to $20 \%$ of patients develop PTDM. The condition was previously termed newonset diabetes mellitus, but because prev- alent diabetes before transplantation has not been excluded in most cases, the name PTDM points to the time of diagnosis rather than the time of occur- rence of the disease.

The term prediabetes was introduced by the American Diabetes Association in 2009 to characterize patients who were at an increased risk of developing type 2 diabetes. Prediabetes is defined as moderately increased fasting plasma glucose levels (impaired fasting glucose [IFG], 100-125 $\mathrm{mg} / \mathrm{dl}$ ) and/or impaired glucose tolerance (IGT)fasting

plasma glucose level $<126 \mathrm{mg} / \mathrm{dl}$ and 603 patients, who were followed for

2-hour glucose level 140 to 199 $\mathrm{mg} / \mathrm{dl}$ - after an OGTT. After the implementa- tion of $\mathrm{HbA}_{1 \mathrm{c}}$ tests as a diagnostic criterion for type 2 diabetes, the $\mathrm{HbA}_{1 \mathrm{c}}$ level in the range of $6.1 \%$ to $6.4 \%$ has also been included in the prediabetes concept. The validity of the prediabetes concept to foresee the development of type 2 diabetes in individual patients is vividly debated, and a recent Cochrane review concluded that prediction can be poor, especially in patients in the lower range of the defined parameters. Furthermore, prediabetes can reverse to normality for up to 11 years after diagnosis.

Nevertheless, $>20 \%$ of patients with kidney transplant and prediabetes convert to PTDM within 6 to 8 years after transplantation. ${ }^{4,5}$ Moreover, moderate increments in blood sugar levels may be a risk marker for patient death. One cohort found that IGT in kidneytransplanted patients represented a mortality risk in the same range as did PTDM, while IFG did not. ${ }^{6}$

This does not mean that moderate impairments in glucose homeostasis lead to patient death. It is reasonable to assume that plasma glucose excursions

represent one of many risk markers for cardiovascular disease as we know them from the definition of the metabolic syndrome. These risk markers include hyperglycemia (prediabetes), which may or may not convert into PTDM; increased blood pressure, insulin resistance, dyslipidemia with hypertriglyceridemia, and lowered highdensity lipoprotein cholesterol; as well as prothrombotic and inflammatory mechanisms (see Figure 1).

The study published by Porrini et al. ${ }^{7}$ in this issue demonstrated for the first time that patients diagnosed with prediabetes have an increased risk of fatal or nonfatal cardiovascular disease when followed for up to 8 years. Prediabetes was defined according to both OGTT glucose criteria and $\mathrm{HbA}_{1 \mathrm{c}}$ criteria. The diagnosis of prediabetes was assessed 12 months after transplantation, a time which serves as baseline for the study.

A total of 7 Spanish centers included

mean of 8 years. Glucose metabolism was normal in $57 \%$ of patients, $27 \%$ had prediabetes, and $16 \%$ had PTDM. Patients with prediabetes had twice the risk of fatal or nonfatal cardiovascular events compared with those 


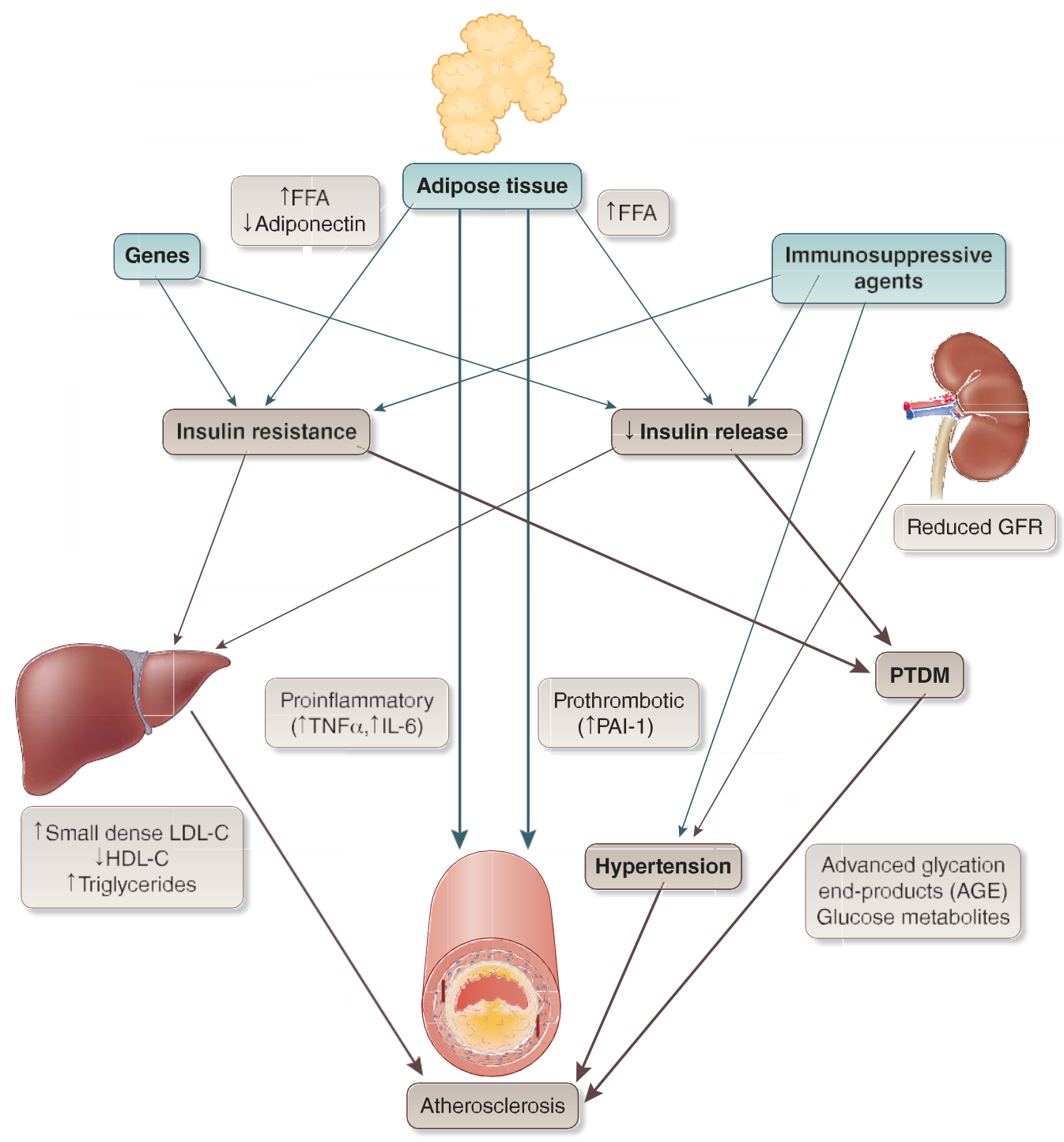

Figure 1 | Mechanisms related to post-transplant diabetes mellitus (PTDM) and atherosclerosis in kidney-transplanted patients. Pathways related to the metabolic syndrome are upregulated in PTDM and prediabetes. Bold arrows point to pathways that are directly related to atherosclerosis. FFA, free fatty acid; GFR, glomerular filtration rate; HDL-C, high-density lipoprotein cholesterol; IL-6, interleukin-6; LDL-C, lowdensity lipoprotein cholesterol; PAI-1, plasminogen activator inhibitor-1 (which is produced in both fat cells and endothelial cells);

TNF-a, tumor necrosis factor-a.

with normal glucose measures. The increased risk persisted even after statistical correction for important cardiovascular risk markers at baseline, such as age, hypertension, proteinuria, pretransplant cardiovascular events, cholesterol, lipid-lowering treatment, and use of angiotensin-converting enzyme inhibitors or angiotensin II receptor blockers. Cardiovascular risk was almost the same for patients with pre- diabetes as for those with PTDM in multivariate analysis.

In contrast to this, a study by Valderhaug et al. ${ }^{6}$ which followed a larger cohort for almost the same period of time, was not able to show that prediabetes predisposed for cardiovascular death. However, in that study the total risk of death was increased for patients with IGT and PTDM, but not for patients with IFG. There are a couple of important differences between the 2 studies. First, in the study by Valderhaug et al., prediabetes was assessed 8 to 10 weeks after transplantation and some of these cases may have reverted to normoglycemia during follow-up, whereas in the study by Porrini et al., the diagnosis of prediabetes was established 12 months post-transplant with less likelihood of reversion. Second, the ethnicity of the 2 populations differs; the study by Valderhaug et al. involved primarily whites, whereas the study by Porrini et al. involved primarily patients of Hispanic origin. Furthermore, one may speculate that the impact of prediabetes on outcome may have been slightly overestimated in the study of Porrini et al., because patients with isolated 2-hour glucose level $\$ 200 \mathrm{mg} / \mathrm{dl}$ (which is actually equivalent to diabetes) were included 
in the study as patients with prediabetes because the authors assumed that they would revert to prediabetes later on. Moreover, a common limitation of all epidemiological studies is that the OGTT is performed only once for practical reasons. The World Health Organization definition requires 2 positive tests for diagnostic assessment in individuals, so the true prevalence of prediabetes or PTDM is not accurately determined in large cohort studies.

What can we learn from the study of Porrini et al.? First of all, prediabetes seems to be an important condition that one should be aware of in kidney- transplanted patients. These patients are primarily found by an OGTT and not by $\mathrm{HbA}_{1 \mathrm{c}}$ assessments. $\mathrm{HbA}_{1 \mathrm{c}}$ alone is an easier marker of intermediate or diabetic hyperglycemia than is an OGTT, but it defines a different population. $\mathrm{HbA}_{1 \mathrm{c}}$ alone was not able to predict cardiovas- cular events or deaths in patients with prediabetes, whereas OGTT-derived glucose markers were necessary to reveal patients at risk. Canwe assess the risk in an easier way by just measuring fasting plasma glucose levels to find pa- tients with IFG? The answer is probably no. Of all patients with IFG in the study, 2 of 3 also had IGT. Bearing in mind that IGT (and not IFG) was the risk factor for mortality in the study by Valderhaug et al. ${ }^{6}$ the OGTT is still the criterion standard to find these patients. ${ }^{1}$
How should kidney-transplanted patients with prediabetes be taken care of? According to the current knowledge, glucose-lowering treatment with phar- maceutical agents is not car- dioprotective per se, although promising agents such as sodium glucose transporter-2 inhibitors and glucagon- like peptide1 receptor agonists are being explored in type 2 diabetes. Moreover, no immunosuppressive regimen has been proven more car- dioprotective in these patients than does any other regimen, although conversion from tacrolimus to cyclosporine may reduce the glycemic burden in some patients. ${ }^{8}$ At present, it is fair to say that diagnosing prediabetes serves as a tool to find patients who would benefit from multitargeted cardioprotective treatment, which involves blood pressure control, cholesterol-lowering treatment, as well as glucose-lowering treatment. In this picture, one should not forget that lifestyle changes may have a beneficial impact on all these risk markers. Multitargeted treatment is proven most helpful in patients with type 2 diabetes, because it seems to prolong life more than 8 years in patients followed for 2 decades. ${ }^{9}$ But, most of all, before we can be sure about this, intervention studies must also be performed in patients with kidney transplant and diabetes. Our patients deserve that such studies are carried out.
DISCLOSURE

TGJ has received lecture fees from

AstraZeneca, Shire, Boehringer Ingelheim, and Novo Nordisk.

\section{REFERENCES}

1. Sharif A, Hecking $M$, deVries $A P$, et al. Proceedings from an international consensus meeting on posttransplantation diabetes mellitus: recommendations and future directions. Am J Transplant. 2014;14:1992-2000.

2. American Diabetes Association. Diagnosis and classification of diabetes mellitus. Diabetes Care. 2011;34:S62-S69.

3. Richter B, Hemmingsen B, Metzendorf MI, Takwoingi Y. Development of type 2 diabetes mellitus in people with intermediate hyperglycaemia. Cochrane Database Syst Rev. 2018;10:CD012661.

4. Hagen M, Hjelmesaeth J, Jenssen T, et al. A 6year prospective study on new onset diabetes mellitus, insulin release and insulin sensitivity in renal transplant recipients. Nephrol Dial Transplant. 2003;18:2154-2159.

5. Porrini EL, Diaz JM, Moreso F, et al. Clinical evolution of post-transplant diabetes mellitus. Nephrol Dial Transplant. 2016;31:495-505.

6. Valderhaug TG, Hjelmesæth J, Hartmann A, et al. The association of early post-transplant glucose levels with long-term mortality. Diabetologia. 2011;54:1341-1349.

7. Porrini E, Díaz JM, Moreso F, et al. Prediabetes is a risk factor for cardiovascular disease following renal transplantation. Kidney Int. 2019;96:1374-1380.

8. Wissing KM, Abramowicz D, Weekers L, et al Prospective randomized study of conversion from tacrolimus to cyclosporine A to improve glucose metabolism in patients with posttransplant diabetes mellitus after renal transplantation. Am J Transplant. 2018;18: 1726-1734.

9. Gaede P, Oellgaard J, Carstensen B, et al. Years of life gained by multifactorial intervention in patients with type 2 diabetes mellitus and microalbuminuria: 21 years follow-up on the Steno-2 randomised trial. Diabetologia. 2016;49:2298-2307. 